\title{
Atuação de nutricionistas responsáveis técnicos pela alimentação escolar de municípios de Minas Gerais e Espírito Santo
}

\author{
Actions of nutritionists as the technicians responsible for school food \\ in municipalities in the states of Minas Gerais and Espírito Santo
}

Anelise Andrade de Souza ${ }^{1}$

Olívia Maria de Paula Alves Bezerra ${ }^{2}$

Elido Bonomo ${ }^{3}$

Camilo Adalton Mariano da Silva ${ }^{3}$

${ }^{1}$ Departamento de Alimentos, Escola de Nutrição, Universidade Federal de Ouro Preto (UFOP). Morro do

Cruzeiro, Campus Universitário. 35400-000 Ouro Preto MG Brasil. asouzandrade@ yahoo.com.br

${ }^{2}$ Escola de Medicina, UFOP. Ouro Preto MG Brasil.

${ }^{3}$ Departamento de Nutrição Clínica e Social, Escola de Nutrição, UFOP. Ouro Preto MG Brasil.

\begin{abstract}
The nutritionist is responsible for promoting eating and nutritional security in school food, thereby contributing to the adoption of healthy eating habits. The scope of this study was to assess the actions of nutritionists as the technicians responsible for school food in the states of Minas Gerais and Espirito Santo. A cross-sectional study was conducted with the participation of 77 nutritionists, and included descriptive, univariate, multivariate and Poisson regression analysis with robust variance. The results revealed non-compliance by the majority of nutritionists with various fundamental attributions of the program as required by law. These include the compilation of the annual work plan, analysis of the nutritional composition of menus, elaboration of technical procedures for food preparation, use of the Good Preparation Practices Manual and conducting the test of acceptability. The regression models showed that nutritionists who prepared a work plan, performed nutritional assessment and conducted tests of acceptability, perform more Food and Nutrition Education actions. These findings highlight the urgent need to bring the municipalities into line with prevailing legislation and implement corrective actions.
\end{abstract}

Key words Nutritionist, Performance. School feeding
Resumo O nutricionista é responsável pela promoção da segurança alimentar e nutricional na alimentação escolar, contribuindo para a construção de práticas alimentares saudáveis. Neste estudo buscou-se conhecer a atuação de nutricionistas responsáveis técnicos da alimentação escolar em municípios de Minas Gerais e Espírito Santo. Foi realizado estudo de corte transversal com participação de 77 nutricionistas, incluindo análises descritivas; univariadas e análises de regressão de Poisson com variância robusta. Constatou-se o descumprimento pela maioria dos nutricionistas de diversas atribuições essenciais do programa, previstas em Lei, como produção do plano anual de trabalho, análise da composição nutricional de cardápios, elaboração de fichas técnicas de preparação, utilização do Manual de Boas Práticas de Fabricação e realização do teste de aceitabilidade. Os modelos de regressão mostraram que nutricionistas que desenvolviam plano de trabalho, avaliação nutricional e teste de aceitabilidade apresentaram maior ocorrência de realização de ações de Educação Alimentar e Nutricional. Ressalta-se necessidade imediata de adequação dos municípios à legislação vigente e implementação de ações corretivas.

Palavras-chave Nutricionista, Atuação, Alimentação Escolar 


\section{Introdução}

O nutricionista é um profissional generalista da área da saúde responsável pela promoção da segurança alimentar e atenção nutricional em todos os seus campos de atuação, contribuindo na manutenção e recuperação do estado nutricional de indivíduos e coletividades ${ }^{1-3}$.

Com a regulamentação da profissão em 1967 houve um grande aumento dos cursos de graduação no Brasil e, em consequência, do número de profissionais em campo, o que propiciou o desenvolvimento de várias áreas de atuação deste profissional $^{4-6}$.

No Programa Nacional de Alimentação Escolar (PNAE), o nutricionista vem ganhando destaque na medida em que promove hábitos alimentares saudáveis, respeitando a cultura alimentar local, na perspectiva da construção da Segurança Alimentar e Nutricional (SAN) ${ }^{7}$. Mudanças no aparato legal do programa resultaram em avanços significativos, dentre eles a crescente contratação de nutricionistas, consolidando este profissional como Responsável Técnico (RT) pelas ações de alimentação escolar ${ }^{7}$.

Resoluções do Conselho Federal de Nutricionistas (CFN) publicadas a partir de $2005^{8,9}$ orientam e normatizam as atividades que cabem aos nutricionistas que atuam no PNAE. A Lei ${ }^{\circ}$ $11.947 / 2009^{10}$ define o nutricionista como o profissional RT pelo programa, devendo o mesmo estar obrigatoriamente vinculado ao setor de alimentação da Entidade Executora (EE) e cadastrado como RT no Fundo Nacional de Desenvolvimento da Educação (FNDE) ${ }^{10}$.

Compete ao nutricionista que atua na alimentação escolar realizar o diagnóstico e o acompanhamento do estado nutricional de todos os escolares e efetuar o planejamento e o acompanhamento de todas as etapas que se referem à elaboração do cardápio até a chegada do alimento adequado à mesa dos estudantes ${ }^{11}$.

A ele cabe, também, a coordenação das ações de alimentação escolar, a inserção nos cardápios de produtos da agricultura familiar respeitando os hábitos alimentares, a vocação agrícola e a cultura alimentar de cada região e a realização de ações de Educação Alimentar e Nutricional (EAN) nas escolas atendidas pelo programa ${ }^{10}$.

O presente estudo teve como objetivo caracterizar o nutricionista RT pelo PNAE utilizando alguns indicadores de condições socioeconômicas e demográficas e conhecer a sua atuação em municípios de Minas Gerais e Espírito Santo, segundo as normativas do programa ${ }^{9,10}$.

\section{Métodos}

Trata-se de um estudo de corte transversal com amostra não probabilística, utilizando dados coletados em entrevista presencial para aplicação de questionário semiestruturado aos nutricionistas RTs pelos municípios. A coleta de dados ocorreu durante a realização de atividades de formação desenvolvidas por um Centro Colaborador em Alimentação e Nutrição do Escolar (CECANE) em 77 municípios de Minas Gerais e Espírito Santo, no período de agosto de 2009 a dezembro de 2013.

Esses municípios foram selecionados pelo FNDE para participação nas atividades do CECANE, a partir dos seguintes critérios de inclusão, considerados simultaneamente: municípios com acesso à internet para a implantação do software SIM PNAE (Sistema de Monitoramento do PNAE); municípios visitados pelo setor de monitoramento do PNAE/FNDE nos anos de 2009 a 2011; municípios relacionados no $33^{\circ}$ Sorteio Público de Municípios da Controladoria Geral da União (CGU) e municípios com nutricionistas cadastrados no FNDE.

O questionário aplicado contemplou aspectos socioeconômicos, demográficos, das condições de trabalho e da atuação dos nutricionistas RT no setor de alimentação escolar. As respostas foram utilizadas na construção de um banco de dados utilizado neste estudo, cuja análise foi iniciada em 2014. Os dados obtidos, sistematizados no Programa Microsoft Excel $2007^{12}$ e duplamente digitados, foram analisados por meio do software STATA versão $12.0^{13}$.

As regulamentações consideradas para avaliar a atuação do nutricionista RT do município foram a Lei $\mathrm{n}^{0} 11.947 / 2009^{10}$, a Resolução/CD/ FNDE n $38 / 2009^{11}$, além das Resoluções do Conselho Federal de Nutricionistas, Resolução/CFN $n^{\circ} 358 / 2005^{8}$ e Resolução/CFN n ${ }^{\circ}$ 465/2010 .

As variáveis dependentes escolhidas para serem analisadas no estudo foram Ações de Educação Alimentar e Nutricional ${ }^{10,11}$ e Compra dos produtos da agricultura familiar pela EE municipal $^{10,11}$, por serem duas grandes conquistas do programa, tornadas obrigatórias às EEs conforme dispõe a Lei $n^{0} 11.947 / 2009^{10}$. As variáveis independentes utilizadas foram Produção do plano anual de trabalho ${ }^{8,9}$, Realização de avaliação $n u$ tricional dos alunos ${ }^{8,9,11}$, Oferecimento de alimentação diferenciada aos alunos com necessidades especiais ${ }^{8,9,11}$, Análise da composição nutricional de cardápios $^{8,9,11}$, Elaboração de fichas técnicas de preparação de alimentos ${ }^{8,9}$, Realização de teste de 
aceitabilidade ${ }^{11}$, Frequência regular de visitas técnicas às escolas da zona urbana ${ }^{8,9}$ Frequência regular de visitas técnicas às escolas da zona rural ${ }^{8,9}$, Utilização do Manual de Boas Práticas de Fabricação de Alimentos ${ }^{8,9}$, Frequência regular de cursos de capacitação para manipuladores de alimentos ${ }^{10}$, Participação da articulação com agricultores familiares $^{11}$, Participação no processo de compra via chamada pública, ${ }^{9,11}$ e Participação no processo de compra via licitaçãa ${ }^{8,9,11}$. Para a variável dependente Compra dos produtos da agricultura familiar pela EE municipal ${ }^{10,11}$ também se utilizou como variável independente Ações de Educação Alimentar e Nutricional ${ }^{10,11}$.

Inicialmente foram realizadas análises descritivas das variáveis estudadas para caracterização da amostra, seguidas por análises univariadas entre as variáveis independentes e as dependentes "Ações de Educação Alimentar e Nutricional" e "Compra dos produtos da agricultura familiar pela Entidade Executora (EE) municipal”, utilizando-se o teste do qui-quadrado de Pearson e considerando significativos valores de "p" $<0,20$. Em seguida, para cada variável dependente, foram realizadas análises multivariadas sucessivas, pela regressão de Poisson com variância robusta, entre estas e as variáveis independentes que apresentaram valor de "p" significativo, de tal forma que, após cada etapa multivariada, foram excluídas as variáveis com valor de "p" maior que 0,05. Desta forma, as análises multivariadas foram repetidas até permanecerem, no modelo explicativo final, somente aquelas variáveis independentes que obtiveram valor de "p" menor que 0,05 e intervalo de confiança de 95\%. A análise estatística utilizando o modelo de regressão de Poisson com variância robusta foi escolhida por se tratar de um estudo de corte transversal.

O projeto de pesquisa foi aprovado por Comitê de Ética em Pesquisa da Universidade Federal de Ouro Preto/MG.

\section{Resultados}

Participaram do estudo todos os 77 nutricionistas RTs pelo Programa de Alimentação Escolar (PAE) que estiveram presentes nas ações do CECANE, correspondendo a 77 municípios, sendo $71(92,2 \%)$ de Minas Gerais e 06 (7,8\%) do Espírito Santo.

Predominaram entre eles pessoas do sexo feminino, com menos de cinco anos de conclusão do curso de graduação em nutrição, que haviam realizado cursos de pós-graduação na área clíni- ca, em contrato temporário no município onde atuam como RT e com faixa salarial de 01 a 03 salários mínimos (SM). A Tabela 1 apresenta alguns aspectos socioeconômicos e demográficos dos nutricionistas participantes do estudo.

Dos municípios, $82 \%(\mathrm{n}=63)$ apresentaram mais de 500 alunos, e somente 53,9\% ( $\mathrm{n}=48)$ deles relataram a existência de quadros técnicos (QT) em seus municípios. O quadro técnico é constituído pelos demais nutricionistas que atuam no programa sob a coordenação e supervisão do RT, assumindo com este a responsabilidade solidária $^{8,9}$. Além disso, dos 29 nutricionistas cujos municípios não tinham QT, 28 (96,5\%)

Tabela 1. Caracterização das condições socioeconômicas e demográficas dos nutricionistas responsáveis técnicos pelo PNAE em municípios de MG e ES, 2009 a $2013(n=77)$

\begin{tabular}{|c|c|c|}
\hline Variáveis & $\mathbf{N}$ & $\%$ \\
\hline \multicolumn{3}{|l|}{ Sexo } \\
\hline Feminino & 69 & 89,6 \\
\hline Masculino & 08 & 10,4 \\
\hline \multicolumn{3}{|l|}{$\begin{array}{l}\text { Tempo de conclusão da graduação em } \\
\text { nutrição }\end{array}$} \\
\hline$\leq \mathrm{a} 05$ anos & 50 & 64,9 \\
\hline$>05$ a 10 anos & 21 & 27,3 \\
\hline > a 10 anos & 06 & 7,8 \\
\hline \multicolumn{3}{|l|}{ Realização de cursos de pós-graduação } \\
\hline $\operatorname{Sim}$ & 46 & 59,7 \\
\hline Não & 31 & 40,3 \\
\hline \multicolumn{3}{|l|}{ Área do curso de pós-graduação } \\
\hline Clínica & 32 & 68,8 \\
\hline $\begin{array}{l}\text { Gestão de Unidades de Alimentação e } \\
\text { Nutrição }\end{array}$ & 13 & 28,6 \\
\hline Saúde do Escolar & 01 & 2,6 \\
\hline \multicolumn{3}{|l|}{ Primeiro emprego } \\
\hline Sim & 34 & 44,2 \\
\hline Não & 43 & 55,8 \\
\hline \multicolumn{3}{|l|}{ Tipo de vínculo empregatício } \\
\hline Concursado & 32 & 41,5 \\
\hline Contratado temporariamente & 39 & 50,6 \\
\hline Não informado & 06 & 7,9 \\
\hline \multicolumn{3}{|l|}{ Atuação em outro município } \\
\hline $\operatorname{Sim}$ & 70 & 90,7 \\
\hline Não & 07 & 9,3 \\
\hline \multicolumn{3}{|l|}{ Faixa salarial } \\
\hline s a 01 salário mínimo & 06 & 7,9 \\
\hline > 01 a 03 salários mínimos & 50 & 64,9 \\
\hline > a 03 salários mínimos & 21 & 27,2 \\
\hline
\end{tabular}

Nota: A carga horária semanal média de trabalho dos nutricionistas foi de 25 horas ( \pm 10 ), a média de tempo em exercício no cargo foi de 31,4 meses $( \pm 21,12)$ e a média salarial foi de 2,8 salários mínimos $( \pm 1,19)$. 
atuavam também em outros municípios como RT do PNAE. Percentuais elevados de inadequação das atividades dos nutricionistas nos municípios foram observados em relação à produção de plano anual de trabalho ${ }^{8,9}$, análise da composição nutricional dos cardápios ${ }^{8,911}$, elaboração de fichas técnicas de preparação de alimentos 8 , realização de testes de aceitabilidade ${ }^{11} \mathrm{e}$ utilização do Manual de Boas Práticas de Fabricação de Alimentos $^{8,9}$. A atuação dos nutricionistas RTs pelo PNAE de acordo com as principais atividades previstas pelo programa é descrita na Tabela 2.

Tabela 2. Atuação dos nutricionistas responsáveis técnicos pelo PNAE em municípios de MG e ES, 2009 a 2013 $(\mathrm{n}=77)$.

\begin{tabular}{|c|c|c|c|c|c|}
\hline Variáveis & $\mathbf{N}$ & $\%$ & Variáveis & $\mathbf{N}$ & $\%$ \\
\hline Produção do Plano Anual de Trabalho & & & Frequência de visitas técnicas às escolas da & & \\
\hline $\operatorname{Sim}$ & 23 & 29,8 & zona rural & & \\
\hline Não & 50 & 64,9 & Diária & 00 & - \\
\hline Não informado & 04 & 5,3 & Semanal & 15 & 19,4 \\
\hline Realização de Avaliação Nutricional dos & & & Quinzenal & 14 & 18,2 \\
\hline alunos & & & Mensal & 12 & 15,5 \\
\hline Sim & 47 & 61,0 & Semestral & 04 & 5,2 \\
\hline Não & 26 & 33,7 & Anual & 01 & 1,3 \\
\hline & & & Não realiza visitas & 06 & 7,8 \\
\hline Não informado & 04 & 5,3 & Sem frequencia definida & 07 & 9,0 \\
\hline Oferecimento de alimentação diferenciada & & & Não informado & 18 & 23,6 \\
\hline aos alunos com necessidades especiais & & & Utilização do Manual de Boas Práticas de & & \\
\hline Sim & 43 & 55,8 & Fabricação de Alimentos & & \\
\hline Não & 08 & 10,4 & $\operatorname{Sim}$ & 23 & 29,8 \\
\hline Não possui alunos com necessidades & 15 & 19,4 & Não & 46 & 59,7 \\
\hline especiais & & & Não informado & 08 & 10,5 \\
\hline Não informado & 11 & 14,4 & Frequência de cursos de capacitação para & & \\
\hline Análise da Composição Nutricional dos & & & manipuladores de alimentos & & \\
\hline cardápios & & & Mensal & 03 & 3,9 \\
\hline $\operatorname{Sim}$ & 31 & 40,2 & Semestral & 25 & 32,4 \\
\hline Não & 43 & 55,8 & Anual & 26 & 33,7 \\
\hline Não informado & 03 & 4,0 & Não informado & 23 & 30,0 \\
\hline Elaboração de fichas técnicas de preparação & & & Ações de Educação Alimentar e Nutricional & & \\
\hline dos alimentos & & & $\operatorname{Sim}$ & 51 & 66,2 \\
\hline $\operatorname{Sim}$ & 34 & 44,0 & Não & 22 & 28,5 \\
\hline Não & 39 & 50,6 & Não informado & 04 & 5,3 \\
\hline Não informado & 04 & 5,4 & Participação da articulação com agricultores & & \\
\hline Realização do Teste de Aceitabilidade & & & familiares & & \\
\hline Sim & 33 & 42,8 & Sim & 46 & 59,7 \\
\hline Não & 39 & 50,6 & Não & 18 & 23,3 \\
\hline Não informado & 05 & 6,6 & Não informado & 13 & 17,0 \\
\hline $\begin{array}{l}\text { Frequência de visitas técnicas às escolas da } \\
\text { zona urbana }\end{array}$ & & & $\begin{array}{l}\text { Participação no processo de compra via } \\
\text { chamada pública }\end{array}$ & 54 & 70,2 \\
\hline Diária & 12 & 15,5 & $\operatorname{Sim}$ & 10 & 12,9 \\
\hline Semanal & 26 & 33,7 & Não & 13 & 16,9 \\
\hline Quinzenal & 11 & 14,2 & Não informado & & \\
\hline Mensal & 10 & 12,9 & Participação no processo de compra via & & \\
\hline Semestral & 04 & 5,2 & licitação & 51 & 66,2 \\
\hline Anual & 00 & - & Sim & 15 & 19,4 \\
\hline Não realiza visitas & 04 & 5,2 & Não & 11 & 14,4 \\
\hline Sem frequência definida & 06 & 7,7 & Não informado & & \\
\hline \multirow[t]{3}{*}{ Não informado } & 04 & 5,6 & Município compra da Agricultura Familiar & & \\
\hline & & & $\operatorname{Sim}$ & 60 & 77,8 \\
\hline & & & Não & 17 & 22,2 \\
\hline
\end{tabular}


A Tabela 3 mostra as variáveis independentes que apresentaram associação estatística no teste de qui-quadrado de Pearson $(\mathrm{p}<0,20)$ e foram incluídas no modelo de regressão utilizado no estudo. Para as variáveis dependente Ações de Educação Alimentar e Nutricional ${ }^{10,11}$ e Compra dos produtos da agricultura familiar pela entidade executora municipal ${ }^{10,11}$ a maioria das variáveis

Tabela 3. Associação entre variáveis independentes utilizadas no teste de qui-quadrado de Pearson e variáveis dependentes "Ações de Educação Alimentar e Nutricional" e "Compra dos produtos da agricultura familiar pela Entidade Executora (EE) municipal".

\begin{tabular}{|c|c|c|c|}
\hline \multirow{2}{*}{$\begin{array}{c}\text { "Ações de Educação Alimentar } \\
\text { e Nutricional" }\end{array}$} & \multicolumn{2}{|c|}{ Amostra } & \multirow[b]{2}{*}{$\mathbf{p}$} \\
\hline & $\mathbf{N}$ & $\%$ & \\
\hline \multicolumn{4}{|l|}{ Produção do Plano Anual de Trabalho } \\
\hline $\operatorname{Sim}$ & 23 & 31,5 & 0,005 \\
\hline Não & 50 & 68,5 & \\
\hline \multicolumn{4}{|l|}{ Realização de Avaliação Nutricional dos alunos } \\
\hline $\operatorname{Sim}$ & 47 & 64,4 & $<0,05$ \\
\hline Não & 26 & 35,6 & \\
\hline \multicolumn{4}{|c|}{ Oferecimento de alimentação diferenciada aos alunos com necessidades especiais } \\
\hline Sim & 43 & 84,3 & 0,807 \\
\hline Não & 08 & 15,7 & \\
\hline \multicolumn{4}{|l|}{ Análise da Composição Nutricional dos cardápios } \\
\hline $\operatorname{Sim}$ & 31 & 41,9 & 0,100 \\
\hline Não & 43 & 58,1 & \\
\hline \multicolumn{4}{|l|}{ Elaboração de fichas técnicas de preparação dos alimentos } \\
\hline $\operatorname{Sim}$ & 34 & 46,5 & 0,035 \\
\hline Não & 39 & 53,5 & \\
\hline \multicolumn{4}{|l|}{ Realização do Teste de Aceitabilidade } \\
\hline $\operatorname{Sim}$ & 33 & 45,8 & $<0,05$ \\
\hline Não & 39 & 54,2 & \\
\hline \multicolumn{4}{|l|}{ Frequência regular de visitas técnicas às escolas da zona urbana } \\
\hline $\operatorname{Sim}$ & 49 & 67,0 & 0,078 \\
\hline Não & 24 & 33,0 & \\
\hline \multicolumn{4}{|l|}{ Frequência regular de visitas técnicas às escolas da zona rural } \\
\hline $\operatorname{Sim}$ & 29 & 49,0 & 0,400 \\
\hline Não & 30 & 51,0 & \\
\hline \multicolumn{4}{|l|}{ Utilização do Manual de Boas Práticas de Fabricação de Alimentos } \\
\hline $\operatorname{Sim}$ & 23 & 33,4 & 0,536 \\
\hline Não & 46 & 66,6 & \\
\hline \multicolumn{4}{|c|}{ Frequência regular de cursos de capacitação para manipuladores de alimentos } \\
\hline Sim & 03 & 5,5 & 0,673 \\
\hline Não & 51 & 94,5 & \\
\hline \multicolumn{4}{|l|}{ Participação da articulação com agricultores familiares } \\
\hline Sim & 46 & 71,8 & 0,017 \\
\hline Não & 18 & 28,2 & \\
\hline \multicolumn{4}{|l|}{ Participação no processo de compra via chamada pública } \\
\hline Sim & 54 & 84,4 & 0,036 \\
\hline Não & 10 & 15,6 & \\
\hline \multicolumn{4}{|l|}{ Participação no processo de compra via licitação } \\
\hline $\operatorname{Sim}$ & 51 & 77,3 & 0,015 \\
\hline Não & 15 & 22,7 & \\
\hline \multicolumn{4}{|l|}{ Compra dos produtos da agricultura familiar pela EE municipal } \\
\hline $\operatorname{Sim}$ & 60 & 80,0 & 0,649 \\
\hline Não & 17 & 20,0 & \\
\hline
\end{tabular}


Tabela 3. continuação

\begin{tabular}{|c|c|c|c|}
\hline \multirow{2}{*}{$\begin{array}{c}\text { "Compra dos produtos da agricultura } \\
\text { familiar pela EE municipal" }\end{array}$} & \multicolumn{3}{|c|}{ Amostra } \\
\hline & $\mathbf{N}$ & $\%$ & $\mathbf{p}$ \\
\hline \multicolumn{4}{|l|}{ Produção do Plano Anual de Trabalho } \\
\hline Sim & 23 & 31,5 & 0,171 \\
\hline Não & 50 & 68,5 & \\
\hline \multicolumn{4}{|l|}{ Realização de Avaliação Nutricional dos alunos } \\
\hline $\operatorname{Sim}$ & 47 & 64,4 & 0,294 \\
\hline Não & 26 & 35,6 & \\
\hline \multicolumn{4}{|c|}{ Oferecimento de alimentação diferenciada aos alunos com necessidades especiais } \\
\hline $\operatorname{Sim}$ & 43 & 84,3 & 0,148 \\
\hline Não & 08 & 15,7 & \\
\hline \multicolumn{4}{|l|}{ Análise da Composição Nutricional dos cardápios } \\
\hline Sim & 31 & 41,9 & 0,572 \\
\hline Não & 43 & 58,1 & \\
\hline \multicolumn{4}{|l|}{ Elaboração de fichas técnicas de preparação dos alimentos } \\
\hline $\operatorname{Sim}$ & 34 & 46,5 & 0,147 \\
\hline Não & 39 & 53,5 & \\
\hline \multicolumn{4}{|l|}{ Realização do Teste de Aceitabilidade } \\
\hline Sim & 33 & 45,8 & 0,035 \\
\hline Não & 39 & 54,2 & \\
\hline \multicolumn{4}{|l|}{ Frequência regular de visitas técnicas às escolas da zona urbana } \\
\hline \multicolumn{4}{|l|}{$\operatorname{Sim}$} \\
\hline \multirow[t]{2}{*}{ Não } & 49 & 67,0 & 0,460 \\
\hline & 24 & 33,0 & \\
\hline \multicolumn{4}{|l|}{ Frequência regular de visitas técnicas às escolas da zona rural } \\
\hline Sim & 29 & 49,0 & 0,352 \\
\hline Não & 30 & 51,0 & \\
\hline \multicolumn{4}{|l|}{ Utilização do Manual de Boas Práticas de Fabricação de Alimentos } \\
\hline $\operatorname{Sim}$ & 23 & 33,4 & 0,902 \\
\hline Não & 46 & 66,6 & \\
\hline \multicolumn{4}{|c|}{ Frequência regular de cursos de capacitação para manipuladores de alimentos } \\
\hline Sim & 03 & 5,5 & 0,127 \\
\hline Não & 51 & 94,5 & \\
\hline \multicolumn{4}{|l|}{ Participação da articulação com agricultores familiares } \\
\hline $\operatorname{Sim}$ & 46 & 71,8 & 0,136 \\
\hline Não & 18 & 28,2 & \\
\hline
\end{tabular}

independentes se mostrou significativa nesse teste, constituindo importantes indícios da importância da atuação do nutricionista RT segundo as normativas do programa.

A Tabela 4 apresenta o modelo final de regressão de Poisson com variância robusta utilizando a variável dependente Ações de Educação Alimentar e Nutricional ${ }^{10,11}$. O modelo identificou associação estatística $(\mathrm{p}<0,05)$ com as variáveis independentes Produção de Plano Anual de Trabalho ${ }^{8,9}$, Realização de avaliação nutricional dos alunos $^{8,9,11}$ e Realização de teste de aceitabilidade ${ }^{11}$.

Já para a variável dependente Compra dos produtos da agricultura familiar pela entidade exe- cutora municipal ${ }^{10,11}$, a regressão de Poisson não identificou variáveis independentes que explicassem o modelo e que fossem estatisticamente significativas.

\section{Discussão}

O presente estudo constatou o descumprimento, pela maioria dos nutricionistas participantes, de diversas atribuições essenciais do PNAE previstas na legislação pertinente do programa, sendo elas a Lei $n^{\circ} 11.947 / 2009^{10}$, a Resolução/CD/FNDE $\mathrm{n}^{\circ} 38 / 2009^{11}$ e as Resoluções do $\mathrm{CFN}^{8,9}$ vigentes 
Tabela 4. Modelo final de Regressão de Poisson com variância robusta com valores de RP bruta e ajustada, com intervalo de confiança de 95\%.

\begin{tabular}{lrccccc}
\hline \multicolumn{6}{c}{ “Ações de Educação Alimentar e Nutricional” e variáveis independentes associadas } \\
\hline \multicolumn{1}{c}{ Variável } & RP bruta & p & IC (95\%) & RP ajust & p & IC (95\%) \\
& & & & & & \\
\hline Produção de Plano de Trabalho & 1,33 & 0,011 & $0,02-0,63$ & 4,13 & 0,022 & $1,22-13,94$ \\
Realização de Avaliação Nutricional dos alunos & 13,84 & 0,000 & $4,14-46,90$ & 3,86 & 0,001 & $1,68-8,85$ \\
Realização de Teste de Aceitabilidade & 1,11 & 0,001 & $0,02-0,42$ & 2,88 & 0,047 & $1,96-8,60$ \\
\hline
\end{tabular}

à época da coleta/análise dos dados do estudo. Entre as atribuições descumpridas pode-se citar a produção do plano anual de trabalho ${ }^{8,9}$, a análise da composição nutricional dos cardápios ${ }^{8,9,11}$, a elaboração de fichas técnicas de preparação $0^{8,9}$, a utilização do Manual de Boas Práticas de Fabricação de Alimentos ${ }^{8,9}$ e a realização de teste de aceitabilidade $^{11}$, entre outras condições inadequadas, indicando situação que demanda intervenção imediata.

Foi verificado que a grande maioria dos nutricionistas atuantes na alimentação escolar dos municípios estudados eram do sexo feminino, concordando com os achados de outros auto$\mathrm{res}^{7,14}$. Além disso, apresentavam, em sua maioria, menos de cinco anos de formados, resultado semelhante ao encontrado por Rodrigues et al. $(58,5 \%)^{15}$.

Embora mais da metade dos nutricionistas participantes do presente estudo tenham cursado pós-graduação, a maior parte deles o fez na área de nutrição clínica. Apenas 2,6\% cursaram pós-graduação na área de saúde do escolar ou saúde pública, as quais guardam maior afinidade com as atividades requeridas ao nutricionista na alimentação escolar ${ }^{9,10}$. Tais resultados endossam aqueles encontrados pela pesquisa realizada pelo $\mathrm{CFN}^{16}$ (55,9\%), que estudou a formação complementar de nutricionistas de todo o país e por Mello et al. ${ }^{7}$ (52,2\%), que avaliou o perfil de nutricionistas do PNAE da região nordeste do Brasil. O motivo alegado pela predileção pela área clínica foi o maior número de cursos disponíveis, ao contrário da área de saúde pública e alimentação escolar. Resultado diferente foi encontrado por Letro e Jorge ${ }^{3}$, em que 18,2\% dos nutricionistas do seu estudo realizaram cursos de pós-graduação na área de saúde pública. No entanto, sua pesquisa também conclui que existe uma preferência dos profissionais pela área clínica possivelmente pela maior oferta destes cursos e por serem áreas que empregam maior número de profissionais ${ }^{3}$.

É importante frisar que cursos de formação continuada, sejam eles cursos de pós-graduação ou capacitações, para os nutricionistas que estão atuando em alimentação escolar devem ser priorizados, visto que os cursos de graduação não têm acompanhado o crescimento do trabalho na área, com pouco investimento acadêmico na saúde coletiva, e especificamente na abordagem do PNAE. Cursos de formação continuada são oferecidos pelo FNDE/MEC, em parceria com os CECANEs objetivando a qualificação dos profissionais e sua atualização diante dos novos temas que surgem, podendo ser excelentes ferramentas para a melhoria da atuação do nutricionista ${ }^{7}$.

Outro aspecto que chama a atenção no presente estudo é o fato de que, além de terem pouco tempo de formados e poucos serem especialistas em alimentação escolar ou em saúde coletiva, pouco menos da metade dos nutricionistas estavam no seu primeiro emprego, resultado também semelhante ao encontrado por Scarparo et al. ${ }^{14}(47 \%)$ quando estudou a formação de nutricionistas na região sul do Brasil, e apresentavam, no conjunto, tempo médio de exercício no cargo de apenas 31,4 meses $( \pm 21,12)$. Boog $^{17}$, ao refletir sobre a atuação do nutricionista em saúde pública, relata que o trabalho do nutricionista neste campo parece ser mais atrativo logo nos primeiros anos após a conclusão da graduação, devido ao sentimento de realização profissional que a área proporciona, ao contrário do que acontece, por exemplo, no campo da alimentação coletiva. No entanto, aspectos como a baixa remuneração e a valorização profissional constituem desestímulos importantes para a atuação do nutricionista na área, prejudicando a sua atuação e muitas vezes levando-o a buscar outros caminhos.

Considerando a distribuição dos nutricionistas de acordo com o tipo de vínculo empregatício 
nos municípios estudados, verificou-se que metade deles era contratado por tempo determinado pelas prefeituras municipais, enquanto $41,5 \%$ eram contratados por tempo indeterminado (concursados), e os demais não informaram o tipo de contrato. Esse resultado foi divergente do encontrado por Mello et al. ${ }^{7}$, em seu estudo na região nordeste do país, que identificou apenas $2,9 \%$ dos profissionais contratados temporariamente e $65 \%$ concursados com estabilidade no emprego. Nesse aspecto, chama-se a atenção para a instabilidade provocada pelas formas de contratação temporária. Este tipo de contrato é marcado por relações de vulnerabilidade no emprego, que promovem incertezas tanto para o trabalhador quanto para o serviço, além das relações de trabalho precárias ${ }^{5}$ e dificuldades do profissional em assumir uma postura crítica perante a gestão do programa e o município. Consequentemente, podem gerar resultados insatisfatórios no cumprimento das atribuições do nutricionista e, consequentemente, na qualidade da alimentação escolar ${ }^{7}$.

Acresce-se a tudo isso o fato de que a carga horária média de trabalho, de 25 horas $( \pm 10)$ semanais, não atinge as recomendações das Resoluções do $\mathrm{CFN}^{8,9}$, que estabelecem carga horária média mínima de 30 horas semanais para que o nutricionista RT da alimentação escolar consiga realizar adequadamente todas as suas funções $\mathrm{e}$ atribuições no cargo.

Em relação à composição do QT nos municípios, aproximadamente a metade relatou a inexistência do mesmo, apesar de a maioria deles apresentar número de alunos superior a 500, o que torna necessário, de acordo com a recomendação do $\mathrm{CFN}^{8,9}$, que ocorra a efetiva composição do QT, com carga horária mínima de 30 horas semanais. Além disso, é preocupante o fato de cerca da metade dos municípios com mais de 500 alunos não possuírem QT, conforme recomenda$\mathrm{do}^{8,9} \mathrm{e}$, ao mesmo tempo, o seu único nutricionista RT atuar também no PAE de outro município, pois a sobrecarga de trabalho possivelmente contribui para a não realização de algumas de suas atividades previstas em $\mathrm{Lei}^{10}$.

A esse respeito, embora as Resoluções do $\mathrm{CFN}^{8,9}$, permitam que o nutricionista assuma mais de uma responsabilidade técnica, desde que se observe alguns critérios definidos, os resultados do presente estudo sugerem que a baixa disponibilidade de tempo, aliada às condições precárias e inseguras de contratação e de trabalho dos nutricionistas e a atuação de grande parte deles em outros municípios, possa estar contrariando a legislação vigente ${ }^{9}$, com resultados insatisfatórios no cumprimento de suas atribuições, afetando a realização das atividades previstas. Nesse aspecto, estudo de Chaves et al. ${ }^{18}$ conclui que o cumprimento das atribuições do nutricionista no PNAE relaciona-se diretamente ao quantitativo de profissionais no programa. Assim, não havendo no município o número mínimo de nutricionistas recomendado ${ }^{8,9}$, possivelmente ocorrerá sobrecarga do profissional e descumprimento de parte de suas atribuições ${ }^{18}$.

Outro problema grave verificado diz respeito aos salários dos participantes da pesquisa, já que, ainda que a carga horária semanal média de trabalho seja inferior a 30 horas, $64,9 \%$ recebiam valor entre 1 a 3 SM, e apenas 27,2\% deles recebiam vencimentos superiores a 3 SM para uma carga horária de 40 horas semanais. No outro extremo, chama a atenção o fato de que 7,9\% dos nutricionistas recebiam o equivalente a apenas 1 SM para atuar como responsáveis técnicos do PNAE, com carga horária de até 30 horas semanais, demonstrando, mais uma vez, a precariedade das relações e dos contratos de trabalho vigentes. Esses resultados se aproximam dos encontrados por Mello et $\mathrm{al}^{7}$, em que $64,1 \%$ de nutricionistas da alimentação escolar recebiam de 2 a 5 SM, mas divergem dos achados de Gambardella et al. ${ }^{19}$, que encontraram 59,1\% de nutricionistas recebendo valores acima de 10 SM para exercer funções na saúde pública, clínica e também em alimentação coletiva. No entanto, este mesmo autor constata que as piores relações entre salário e jornada de trabalho são observadas para os nutricionistas da área de saúde pública.

Em relação às atividades desenvolvidas pelos nutricionistas nos municípios, constatou-se que apenas uma pequena parcela deles elaborava o plano anual de trabalho ${ }^{8,9}$, embora o planejamento das atividades a serem desenvolvidas ao longo do ano letivo constitua a primeira etapa na gestão do programa ${ }^{7-9}$ e seja função indelegável do nutricionista RT, cabendo ao mesmo traçar metas, planejar e organizar as ações para atingir os objetivos propostos e avaliar os seus impactos. Ressalta-se que essa etapa independe, de modo geral, da qualidade da estrutura física e material de trabalho disponibilizada ao nutricionista, estando relacionada principalmente à iniciativa $\mathrm{e}$ capacidade de gestão do profissional. Scarparo et al. ${ }^{14}$ encontraram, em um estudo realizado com nutricionistas RT pela alimentação escolar em municípios do sul do país, que o maior impedimento referido pelos mesmos para a execução de atividades previstas nas legislações do PNAE 
se devia à carga horária insuficiente de trabalho. No entanto, a inexecução do plano atual de trabalho pelo profissional não se justifica, visto que somente a partir da sua elaboração torna-se possível um planejamento adequado das atividades a serem desenvolvidas no programa, e esta etapa independe de fatores externos ao profissional.

Da mesma forma, a avaliação nutricional dos alunos $^{8,9,11}$, condição essencial para o planejamento de cardápios nutricionalmente adequados a cada faixa etária e à manutenção/recuperação do estado nutricional da população atendida, não era realizado por cerca de 30\% dos nutricionistas. Embora essa etapa do trabalho dependa também de alguns equipamentos de avaliação nutricional, como balança e antropômetro, que podem não estar disponíveis para a utilização do nutricionista, apesar de serem de baixo custo, sabe-se que a parceria com as secretarias de saúde para a vigilância alimentar e nutricional é altamente desejável, constituindo, portanto, a indisponibilidade de equipamentos justificativa insuficiente para a não realização dessa etapa crucial do PNAE.

Do mesmo modo, a promoção da alimentação saudável utilizando parcerias firmadas com os outros profissionais de saúde do município também constitui iniciativa possível e necessária para que se possa fornecer um atendimento amplo, permitindo implementações e intervenções na área de alimentação e nutrição dos escolares ${ }^{17}$. Deve-se, também, buscar parcerias com outros programas desenvolvidos nos municípios que tenham atuação também dentro do ambiente escolar, como por exemplo, o Programa Saúde na Escola (PSE). Esse programa consiste numa política intersetorial entre a saúde e a educação voltada às crianças, adolescentes, jovens e adultos da educação pública brasileira, que tem como principal objetivo a promoção de saúde e educação integral ${ }^{20}$.

Por outro lado, é possível que a grande maioria desses municípios apresente condições adequadas para adquirir equipamentos básicos para a realização da avaliação nutricional dos escolares, uma vez que o Índice de Desenvolvimento Humano (IDH) médio dos municípios que participaram do estudo foi de 0,679 (mínimo $=0,558$; máximo $=0,8)$. Do total de municípios, $58,4 \%(\mathrm{n}=45)$ se encontravam em uma faixa de médio desenvolvimento, 32,4\% ( $\mathrm{n}=25)$ de alto e $1,3 \%(n=1)$ de muito alto. Somente $7,9 \%(n=6)$ municípios apresentaram IDH na faixa de baixo desenvolvimento ${ }^{21}$. No entanto, o cumprimento das atribuições do nutricionista no PNAE relaciona-se ao quantitativo de profis- sionais no programa, sendo que, se os parâmetros numéricos não estiverem adequados, como observado em um percentual expressivo neste estudo, pode ocorrer sobrecarga de trabalho do RT e consequentemente o não cumprimento de todas as suas atribuições, incluindo a avaliação nutricional de todos os alunos da rede pública de ensino ${ }^{18}$.

A respeito da oferta de cardápio especial aos alunos com necessidades nutricionais diferenciadas $^{8,9,11}$, verificou-se que pouco mais da metade dos nutricionistas relataram fazê-lo, em contraposição aos achados de Scarparo et al. ${ }^{14}$ e Mello et al. ${ }^{7}$. Esses autores encontraram respectivamente, menos de $15 \%$ ou raros nutricionistas realizando tal oferta. Esse achado é importante, pois, apesar das dificuldades já relatadas, denota a observância, por mais da metade dos nutricionistas do presente estudo, a essa prerrogativa do programa. No entanto, ainda pode ser considerado distante do ideal, uma vez que tal atenção deveria ser priorizada por todos os RTs, sob o risco de acarretarem danos imediatos à saúde dos escolares com o não atendimento de uma prerrogativa básica e fundamental do programa, que é o tratamento aos alunos segundo o princípio da equidade ${ }^{10}$.

Também foi observado o não cumprimento de diversas outras atribuições essenciais do nutricionista junto ao programa, em especial daquelas que devem subsidiar a elaboração do cardápio da alimentação escolar ${ }^{9,10}$. Análise da composição nutricional dos cardápios ${ }^{8,9,11}$, elaboração de fichas técnicas de preparação de alimentos ${ }^{8,9}$ e a realização de teste de aceitabilidade ${ }^{11}$ eram realizados por menos da metade dos nutricionistas. A esse respeito, Prado e $\mathrm{Abreu}^{22}$ ressaltam a necessidade de realizar testes de aceitabilidade das preparações servidas, visando ao atendimento das preferências e hábitos alimentares da população atendida, além da diferenciação das porções distribuídas aos alunos segundo suas necessidades; avaliação da composição nutricional dos cardápios e elaboração das fichas de preparação dos alimentos ${ }^{22}$, instrumento que auxilia no planejamento das refeições, possibilitando o conhecimento apurado da qualidade nutricional das preparações oferecidas ${ }^{23}$. Danelon et al. ${ }^{24}$ também encontraram diversas inadequações nos cardápios escolares analisados em seu estudo, dentre elas o descumprimento no atendimento das necessidades nutricionais diárias dos alunos em 50\% dos cardápios analisados e o desequilíbrio entre a oferta de energia e dos demais nutrientes ${ }^{24}$. 
Atividades como análise da composição nutricional do cardápio ${ }^{8,9,11}$, elaboração das fichas técnicas de preparação dos alimentos ${ }^{8,9} \mathrm{e}$ realização de testes de aceitabilidade ${ }^{11}$ são importantes e devem obrigatoriamente ser implementadas pelo nutricionista RT da alimentação escolar, pois envolvem diretamente o consumo alimentar e constituem a essência da dietética, área da Nutrição que inclui o planejamento e a preparação de refeições nutricionalmente adequadas ${ }^{25}$. Também se constata, pelos valores de IDH dos municípios estudados, relatados anteriormente, que os mesmos teriam, em sua grande maioria, condições suficientes para disponibilizar infraestrutura minimamente adequada e equipamentos básicos para a realização de atividades essenciais do programa, como avaliação nutricional dos escolares, EAN e testes de aceitabilidade de novas preparações. O fornecimento de infraestrutura técnico-organizacional necessária para o desenvolvimento dessas ações é responsabilidade da $\mathrm{EE}$, visto que os recursos repassados pela esfera federal são para uso exclusivo na compra de gêneros alimentícios. Assim, para que se operacionalize o PNAE cabe aos municípios arcarem com a adequação da infraestrutura mínima, equipamentos e recursos humanos suficientes ${ }^{26}$.

Outro aspecto relevante diz respeito à presença regular do nutricionista nas escolas urbanas e rurais dos municípios ${ }^{8-10}$. Scarparo et al. ${ }^{14}$, por exemplo, constatou que $61 \%$ dos nutricionistas realizavam regularmente visitas tanto às escolas urbanas quanto às rurais dos municípios estudados. Já no presente estudo, embora tenham sido verificadas frequências mais elevadas de visitas diárias, semanais, quinzenas ou mensais nas escolas urbanas, constatou-se que aproximadamente $18 \%$ dos nutricionistas não as realizavam ou não possuíam um planejamento de visitação dessas escolas, ou mesmo não informaram sobre a periodicidade das visitas. Para as escolas da zona rural esse quadro foi ainda pior, pois, embora cerca da metade dos nutricionistas faça visitas semanais, quinzenais ou mensais às escolas rurais, aproximadamente $40 \%$ deles não fazem cronograma de visitações ou não informam sobre a periodicidade das visitas.

Essa situação também colabora para o inadequado desenvolvimento do programa de alimentação escolar nesses municípios, pois a supervisão in loco por parte do nutricionista, prevista nas normativas do programa ${ }^{10,27}$, permite ao profissional a melhor visualização dos problemas, identificando pontos críticos a fim de corrigi-los imediata e adequadamente, além de permitir sua maior aproximação da comunidade escolar. A falta de apoio dos gestores e o desconhecimento dos mesmos em relação ao trabalho do nutricionista, a desvalorização do programa nos municípios, a falta de autonomia do profissional RT e de recursos como transporte para realizar visitas às escolas da zona urbana e rural podem ser impeditivos graves da execução de todas as atribuições que competem a este profissional, impactando na qualidade do atendimento e do programa ${ }^{14}$.

O mesmo ocorre com relação à utilização do Manual de Boas Práticas de Fabricação de Alimentos $^{8,9}$, já que apenas cerca de $30 \%$ dos nutricionistas responderam que o utilizam. Sabe-se que a efetiva aplicação do Manual de Boas Práticas nas unidades escolares é um indicativo de qualidade do serviço prestado e de segurança na oferta da alimentação escolar aos alunos ${ }^{14}$, e sua elaboração e efetiva observância é obrigação de todo nutricionista que assume a RT pela alimentação escolar.

Por outro lado, foi constatado no presente estudo que $70 \%$ dos nutricionistas realizavam cursos de capacitação para os manipuladores de alimentos ${ }^{10}$ que trabalham nas escolas. Embora a realização desses cursos não substitua a produção e a utilização do Manual de Boas Práticas nas cozinhas escolares, os mesmos tornam os manipuladores mais conhecedores dos assuntos tratados, referentes à importância das boas práticas, perigos existentes na produção e como ocorre e podese evitar a contaminação por microorganismos. A capacitação contribui, então, não somente para melhorar a qualidade higiênico-sanitária, mas também para aperfeiçoar os cuidados no preparo dos alimentos nas cozinhas escolares ${ }^{28}$.

Destaca-se, entre as várias transformações ocorridas no PNAE nos últimos anos, a promulgação da Lei $n^{\circ} 11.947 / 2009^{10}$ a qual proporcionou, entre outras, duas grandes conquistas à sociedade brasileira. A primeira delas diz respeito à obrigatoriedade de inclusão das ações de EAN no processo de ensino e aprendizagem, e a segunda à compra direta de alimentos produzidos por agricultores locais/regionais. A EAN objetiva a abordagem do tema alimentação e nutrição perpassando o currículo escolar e o desenvolvimento de práticas saudáveis de vida, na perspectiva da promoção da segurança alimentar e nutricional. O profissional habilitado para desenvolver essa ação é, segundo essa Lei, o nutricionista RT pelo programa, juntamente com os demais que compõem o QT do município. Já a compra direta de alimentos produzidos por agricultores familiares busca a garantia do acesso dos escolares a 
alimentos de melhor qualidade, em consonância com hábitos alimentares, cultura e tradição alimentar local, pautando-se na sustentabilidade e diversificação agrícola da região ${ }^{10}$.

A respeito das ações de EAN, verificou-se que $28,5 \%$ dos participantes do estudo não as desenvolvia, demonstrando descumprimento do que dispõe as normativas do programa ${ }^{10,11,26} \mathrm{e}$ do $\mathrm{CFN}^{8,9}$. Ações educativas em alimentação e nutrição são de grande importância na promoção da saúde e da alimentação saudável. No entanto, reconhece-se que há maior ênfase na informação do que na prática, e que seu papel não está ainda claramente definido, existindo, muitas vezes, uma lacuna entre o discurso e a prática ${ }^{29}$. Sabe-se que a implementação de programas de EAN nas escolas e a consequente promoção de ambientes favoráveis à adoção de práticas alimentares adequadas e saudáveis constituem importantes estratégias para o desenvolvimento pleno das crianças e adolescentes. Além disso, o PNAE deve oferecer todo o suporte necessário para organizar e facilitar o encontro entre o sujeito que aprende e o conhecimento a ser aprendido ${ }^{30-34}$.

A Lei n ${ }^{0} 11.947 / 2009^{10}$ e a Resolução/CD/ FNDE $n^{\circ}$ 38/200911 determinam a participação do nutricionista RT nos processos de articulação de agricultores familiares para o fornecimento de gêneros alimentícios, assim como na compra destes gêneros via chamada pública e via licitação. Verificou-se, no presente estudo, que mais de $60 \%$ deles o faziam, e $77,8 \%$ destes já se encontravam em processo de compra direta dos produtos da agricultura familiar por meio da chamada pública. Esse é um dado bastante positivo, pois indica uma maior atenção do nutricionista para o cumprimento desse item da $\mathrm{Lei}^{10}$, o que pode ser explicado pela obrigatoriedade de compra destes produtos, a responsabilidade técnica do nutricionista em relação ao cumprimento da legislação e as penalidades a que podem ser submetidos os municípios caso a compra desses produtos não aconteça ${ }^{10,11}$.

O modelo de regressão utilizando a variável dependente "Ações de Educação Alimentar e

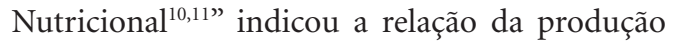
do plano anual de trabalho ${ }^{8,9}$ pelo nutricionista RT; a realização de avaliação nutricional dos alunos $^{8,9,11}$ e do teste de aceitabilidade ${ }^{11}$ das preparações servidas para o cumprimento adequado das atividades básicas do programa. Tais variáveis independentes influenciaram diretamente na realização das atividades de EAN, segundo o modelo final de regressão de Poisson, sendo essa uma das principais premissas do programa.
Quanto à regressão utilizando a variável dependente "Compra de produtos da agricultura familiar pela EE do município ${ }^{10,11}$ ”, não foram encontradas variáveis independentes que se associassem ao modelo, possivelmente devido ao fato de que a obrigatoriedade da implementação da aquisição de produtos da agricultura familiar dentro de prazos determinados pelo $\mathrm{FNDE}^{10}$ tenha levado grande parte dos nutricionistas a privilegiarem esse aspecto da $\mathrm{Lei}^{10} \mathrm{em}$ detrimento de outros.

No entanto, ainda que algumas variáveis independentes não tenham sido significativas no modelo, tais como oferecimento de alimentação diferenciada aos alunos ${ }^{8,9,11}$ com necessidades especiais, avaliação da composição nutricional dos cardápios ${ }^{8,9,11}$, elaboração de fichas técnicas de preparação de alimentos $^{8,9}$, participação na articulação com agricultores familiares ${ }^{11}$ e nos processos de compra via chamada pública ${ }^{9,11}$ e licitação $0^{8,9,11}$, entre outros, destaca-se a importância do cumprimento das demais atribuições do nutricionista relacionadas na legislação do programa, de forma a fornecer um atendimento adequado para a população escolar.

Ressalta-se que se trata de um estudo de delineamento transversal, que tem como característica a dificuldade de se estabelecer a sequência temporal entre uma suposta causa e seu efeito, e distinguir se uma associação é causal ou não. Entretanto sua validade interna não é afetada e seus resultados poderão ser extrapolados para municípios que apresentem cenários semelhantes aos estudados neste trabalho.

\section{Conclusão}

As diretrizes e os princípios do PNAE mostram que a sua execução é complexa e dependente da presença de quantitativos adequados de nutricionistas efetivamente qualificados e comprometidos com o programa nas suas entidades executoras, de forma que seu trabalho resulte na melhoria da qualidade da alimentação e da saúde da população escolar.

Os resultados encontrados indicam a inobservância de princípios básicos do programa, conforme demonstrado, afetando a qualidade/ quantidade da alimentação que é ofertada aos escolares, a elaboração das fichas técnicas de preparação, a análise da composição nutricional e a aceitação e efetiva ingestão por parte dos alunos, o que pode indicar situação de descumprimento das responsabilidades técnicas do nutricionista. 
Ainda que a insuficiência de profissionais ou de tempo dos mesmos para execução de suas funções e falta de infraestrutura básica para o funcionamento do programa possam figurar como determinantes ou condicionantes desse quadro, a limitação da formação do profissional, a carência de programas de capacitação continuada para os nutricionistas em campo e a inexperiência do recém-formado também são fatores que podem resultar no descumprimento das atribuições do RT.

Por outro lado, os resultados do estudo também evidenciaram aspectos positivos na atuação da maioria dos nutricionistas no que diz respeito à participação dos mesmos nos processos de compra dos produtos da agricultura familiar para a alimentação escolar.

Tais achados indicam a necessidade de cumprimento das normativas do programa por parte desses municípios e da adoção de medidas corretivas, a fim de resolver as inadequações identificadas. Nesse aspecto, ressalta-se a importância da adequação dos quadros técnicos, das cargas horárias e das condições de trabalho dos nutricionistas, incluindo a melhoria das relações de trabalho e a efetiva valorização do profissional enquanto gestor e responsável, em nível municipal, por um dos maiores programas de promoção da Segurança Alimentar e Nutricional do país.

Cabe ao poder público, em seus diferentes níveis, propiciar condições humanas, físicas e materiais para o adequado desenvolvimento do PNAE e às universidades propiciarem um currículo escolar que contemple as ações que deverão ser realizadas pelo nutricionista que atuará em saúde coletiva e com o PNAE.

Nesse aspecto, ressalta-se que os resultados aqui relatados foram apropriados pelo CECANE para o planejamento e o desenvolvimento de ações corretivas, entre elas formação continuada, assessoria aos municípios e capacitações de gestores, nutricionistas, conselheiros de alimentação escolar, agricultores familiares, entre outros atores do PNAE.

Reforça-se a importância de ações conjuntas entre os nutricionistas responsáveis técnicos dos municípios com os demais atores do programa, assim como sua interação com os demais membros da comunidade escolar e do poder público local, com vistas a contribuir efetivamente para a garantia do Direito Humano à Alimentação Adequada e Saudável e da Segurança Alimentar e Nutricional do alunado atendido pelo programa.

Constataram-se dificuldades na elaboração e execução da metodologia utilizada nesta pesquisa, devido à insuficiência, na literatura, de estudos de avaliação da atuação do nutricionista Responsável Técnico pelo Programa Nacional de Alimentação Escolar, dificultando a comparação com outros estudos. Sendo assim, observa-se a necessidade de novos estudos nesta área.

\section{Colaboradores}

AA Souza participou da concepção, planejamento e desenho do estudo, análise e interpretação dos dados e redação do artigo. OMPA Bezerra participou do planejamento e desenho do estudo e colaborou na redação do artigo e revisão crítica do mesmo. E Bonomo colaborou na revisão crítica do artigo. CAM Silva participou da concepção, planejamento e desenho do estudo, análise e interpretação dos dados e colaborou na redação e na revisão crítica do artigo. 


\section{Referências}

1. Medeiros MAT, Amparo-Santos L, Domene SMA. Education of dietitian's in Brazil: Minimum clock hours of instruction for a bachelor's degree in Nutrition. Rev Nutr 2013; 26(5):583-593.

2. Pinheiro ARO, Recine E, Alencar B, Fagundes AA, Sousa JS, Monteiro RA, Toral N. Percepção de professores e estudantes em relação ao perfil de formação do nutricionista em saúde pública. Rev Nutr 2012; 25(5):631643.

3. Letro LCMAO, Jorge MN. Inserção profissional dos nutricionistas egressos do Centro Universitário do Leste de Minas Gerais - UNILESTE/MG. Nutrir Gerais 2010; 4(7):668-680.

4. Miranda DEGA, Pereira CHC, Paschoini TB, Quaglio T. O perfil de atuação dos ex alunos do curso de nutrição de uma universidade do interior paulista. Investigação 2010; 10(2-3):54-59.

5. Panice GMN, Gomes A. Atuação profissional dos egressos do curso de nutrição de uma instituição de ensino superior do Paraná. Rev Saúde e Pesquisa 2008; 1(1):4550.

6. Vasconcelos FAG, Calado CLA. Profissão nutricionista: 70 anos de história no Brasil. Rev Nutr 2011; 24(4):605617.

7. Mello AL, Vidal Júnior PO, Sampaio LR, Santos LAS, Freitas MCS, Fontes GAV. Perfil do nutricionista do programa nacional de alimentação escolar na região Nordeste do Brasil. Rev Nutr 2012; 25(1):119-132.

8. Brasil. Conselho Federal de Nutricionistas. Resolução/ CFN n³58 de 18 de maio de 2005. Dispõe sobre as atribuições do Nutricionista no âmbito do Programa de Alimentação Escolar (PAE) e dá outras providencias. Diário Oficial da União 2005; 28 dez.

9. Brasil. Conselho Federal de Nutricionistas. Resolução/ CFN n ${ }^{\circ} 465$ de 23 de agosto de 2010. Dispõe sobre as atribuições do Nutricionista, estabelece parâmetros numéricos mínimos de referencia no âmbito do Programa de Alimentação Escolar (PAE) e dá outras providencias. Diário Oficial da União 2010; 25 ago.

10. Brasil. Lei $\mathrm{n}^{\circ} 11.947$, de 16 de junho de 2009. Dispões sobre o atendimento da alimentação escolar e do programa dinheiro direto na escola aos alunos da educação básica. Diário Oficial da União 2009; 17 jul.

11. Brasil. Resolução/CD/FNDE n³8, de 16 de julho de 2009. Dispõe sobre o atendimento da alimentação escolar aos alunos da educação básica no Programa Nacional de Alimentação Escolar (PNAE). Diário Oficial da União 2009; 17 jul.

12. Microsoft Excel [Programa de computador]. Versão 8.0. Microsoft Seattle, 1997.

13. STATA. Data Analysis and Statistical Software [Programa de computador]. Versão 12.0.

14. Scarparo ALS, Oliveira VR, Bittencourt JMV, Ruiz ENF, Fernandes PF, Zys JZ, Moulin CC. Formação para nutricionistas que atuam no Programa Nacional de Alimentação Escolar: uma avaliação da efetividade. Cien Saude Colet 2013; 18(4):1001-1008.

15. Rodrigues KM, Peres F, Waissmann W. Condições de trabalho e perfil profissional dos nutricionistas egressos da Universidade Federal de Ouro Preto, Minas Gerais, entre 1994 e 2001. Cien Saude Colet 2007; 12(4):1021-1031.
16. Conselho Federal de Nutricionistas (CFN). Inserção profissional dos nutricionistas no Brasil. Brasília: CFN; 2006. [acesso $2014 \mathrm{dez} 01$ ]. Disponível em: http://www. cfn.org.br/novosite/pdf/pesquisa.pdf.

17. Boog MCF. Atuação do nutricionista em saúde pública na promoção da alimentação saudável. Revista Ciência ఓ Saúde 2008; 1(1):33-42.

18. Chaves LG, Santana TCM, Gabriel CG, Vasconcelos FAG. Reflexões sobre a atuação do nutricionista no Programa Nacional de Alimentação Escolar no Brasil. Cien Saude Colet 2013; 18(4):917-926.

19. Gambardella AMD, Ferreira CF, Frutuoso MFP. Situação profissional de egressos de um curso de nutrição. Rev Nutr 2000; 13(1):37-40.

20. Portal da Saúde. Programa Saúde na Escola. [acessado 2015 set 14]. Disponível em: http://dab.saude.gov.br/ portaldab/pse.php

21. Instituto Brasileiro de Geografia e Estatística (IBGE). [acessado 2015 jul 02]. Disponível em: http://www.cidades.ibge.gov.br/

22. Prado SD, Abreu MSD. Nutricionista: onde trabalha? Quais suas condições de trabalho? Rev Nutr 1991; 4(1/2):65-92.

23. Adjafre A, Adjafre R, Boas GF, Dowsley C. Elaboração de ficha técnica de preparação e modificação de preparações com perfil nutricional inadequado oferecidas na merenda de uma escola particular do Distrito Federal. Nutrire 2011; 36:95-95.

24. Danelon MAS, Danelon MS, Silva MV. Alcance das metas nutricionais do programa "Escola de Tempo Integral”, no município de Piracicaba, SP. Segurança Alimentar e Nutricional 2008; 15(1):15-28.

25. Akutsu RC, Botelho RA, Camargo EB, Sávio KEO, Araújo WC. A ficha técnica de preparação como instrumento de qualidade na produção de refeições. Rev Nutr 2005 18(2):277-279.

26. Santos LMP, Santos SMC, Santana LAA, Henrique FCS, Mazza RPD, Santos LAS, Santos LS. Avaliação de políticas públicas de segurança alimentar e combate a fome no período de 1995-2002. 4-Programa Nacional de Alimentação Escolar. Cad Saude Publica 2007; 23(11):2681-2693.

27. Brasil. Resolução/CD/FNDE n²6, de 17 de junho de 2013. Dispõe sobre o atendimento da alimentação escolar aos alunos da educação básica no Programa Nacional de Alimentação Escolar - PNAE. Diário Oficial da União 2013; 17 jun.

28. Saccol ALF, Rubim BA, Mesquita MO, Welter L. Importância de treinamento de manipuladores em boas práticas. Disc. Scientia 2006; 7(1):91-99.

29. Santos LAS. O fazer educação alimentar e nutricional: algumas contribuições para reflexão. Cien Saude Colet 2012; 17(2):453-462.

30. Cervato-Mancuso AM, Silva GM, Cárdenas AP. Grupo focal con nutricionistas que actúan en la alimentación escolar: el menu como elemento pedagógico. Rev Chil Nutr 2013; 40(3):250-255.

31. Albuquerque AG, Pontes CM, Osório MM. Knowledge of educators and dieticians on food and nutrition education in the school environment. Rev Nutr 2013; 26(3):291-300. 
32. Fernandes PS, Bernardo CO, Campos RMMB, Vasconcelos FAG. Avaliação do efeito da educação nutricional na prevalência de sobrepeso/obesidade e no consumo alimentar de escolares do ensino fundamental. J Pediatr (Rio J) 2009; 85(4):315-321.

33. Bettão GV, Lopez MAV, Navarro MLBA. Políticas públicas e sua intervenção na saúde nutricional. Revista Cesumar 2006, 11(1):89-99.

34. Camossa ACA, Costa FNA, Oliveira PF, Figueiredo TP. Educação nutricional: uma área em desenvolvimento. Alim. Nutr. Araraquara 2005; 16(4):349-354.

Artigo apresentado em 09/03/2015

Aprovado em 02/08/2016

Versão final apresentada em 04/08/2016 